\title{
CONSUMER PROTECTION AND THE INCENTIVE TO BECOME INFORMED
}

\author{
Mark Armstrong \\ University College London
}

John Vickers

All Souls College, Oxford

\author{
Jidong Zhou \\ University College London
}

\begin{abstract}
We discuss the impact of consumer protection policies on consumers' incentives to become informed of the best deals available in the market. In a market with costly information acquisition, we find that imposing a cap on suppliers' prices reduces the incentive to become informed of market conditions, with the result that prices paid by consumers (both informed and uninformed) may rise. In a related model where consumers have the ability to refuse to receive marketing, we find that this ability softens price competition and can make all consumers worse off. (JEL: D18, D83, L51)
\end{abstract}

\section{Introduction}

An important determinant of the intensity of competition in some markets is the effort that consumers make to search out good deals and avoid bad deals. If consumers typically compare the offers of very few firms, then the elasticity of demand facing each firm is low, so equilibrium prices will tend to be high, irrespective of the supply-side structure of the market. In the limit where no consumers make price comparisons there is the Diamond Paradox that the equilibrium price is the monopoly price whatever the number of firms. On the other hand, the more that consumers know about deals in the market, the greater is the competitive pressure on firms to offer good deals. Thus there is a positive externality between consumers in that each consumer benefits when others possess better market information. In many cases consumers must incur a private cost to obtain better market information (which might simply comprise the cognitive costs of processing more information). In such circumstances, policies which act

Acknowledgments: We are grateful for comments from Michael Castanheira, Maarten Janssen, José Luis Moraga-González and Ivan Png. The support of the Economic and Social Research Council (UK) is gratefully acknowledged.

E-mail addresses: Armstrong: mark.armstrong@ucl.ac.uk; Vickers: john.vickers@all-souls.ox. ac.uk; Zhou: jidong.zhou@ucl.ac.uk 
indirectly to reduce incentives to acquire information may harm consumers, even if the policies are intended to protect consumers from more direct harm.

We consider two such consumer protection policies: a cap on the prices suppliers can charge, and measures which enable consumers to refuse to receive advertising. A price cap which protects consumers from bad deals may be a mixed blessing. The direct effect of the regulation is positive for consumers because high pricing is prevented. But the policy reduces price dispersion and blunts incentives to become informed about the available prices, which in turn weakens the competitive pressure on firms to offer low prices. Likewise, a policy which allows consumers to opt out of advertising reduces the proportion of consumers who are well-informed about deals in the market, which encourages firms to offer higher prices. This indirect effect might outweigh the direct benefit to those consumers who dislike receiving intrusive marketing.

The market we model is an extension of Burdett and Judd (1983). We allow for a richer information structure than that paper, as well as for (plausible) heterogeneity in consumer information costs. We then use this model to discuss the two consumer protection policies. Fershtman and Fishman (1994) examined the impact of a price cap in Burdett and Judd's model, and showed that the price cap acted to raise expected prices. Thus, the indirect competition-weakening effect of regulation outweighed the direct price-limiting effect (unless regulation is so tight as to remove any incentive to search). In Section 3.1, we revisit their analysis using our extended model. We allow for a richer information structure and find that when information costs are constant across consumers, the price-raising effect of price caps continues to be robustly present. However, when consumers differ in their cost of acquiring better information the impact of a price cap on consumers is ambiguous. (A possible behavioral setting in which consumers differ in their information costs is when an exogenous fraction of consumers are "naive" and mistakenly believe there is no benefit in shopping around.)

Likewise, our analysis in Section 3.2 shows that introducing measures that permit consumers to opt out of advertising has ambiguous effects on consumers. When consumers are alike in their psychological aversion to advertising, though, the impact of the policy is harmful to consumers, and the indirect impact of the policy to relax competition outweighs the direct benefit to ad-averse consumers. This negative impact can be overturned when consumers differ in their disutility from advertising, although the introduction of such measures will harm those consumers who are not strongly ad-averse.

\section{Description of a Market}

A large number of identical firms, $F$ in number, supply a homogeneous product to a continuum of consumers of unit mass. For simplicity, normalize the cost of 
supply to zero. Consumers are risk-neutral, and all have maximum willingnessto-pay for a unit of the product equal to $v$. Consumers are endogenously divided into two groups according to their choice of search technology: the informed (or more informed) and the uninformed (or less informed). The former observe more prices on average than the latter.

Specifically, suppose that the informed use a search technology such that the probability of observing exactly $n \leq F$ distinct prices which is equal to $\alpha_{n}$, where $\sum_{n=0}^{F} \alpha_{n}=1$. Similarly, the less informed use a search technology such that the chance of observing exactly $n$ prices is equal to $\beta_{n}$, where $\sum_{n=0}^{F} \beta_{n}=1$. It is convenient to make the following assumptions on consumer information:

$$
\alpha_{0}=\beta_{0}=0 ; \quad \beta_{1}>0 ; \quad \alpha_{1}=0 .
$$

The first part of equation (1) states that all consumers are aware of at least one price and so can make a purchase. The second part states that less informed consumers have a chance of observing only one price, and this implies that firms have some market power because they may face a consumer with no choice of supplier. The third part states that the more informed consumers always have a choice of supplier, which implies that when all consumers are informed the market is perfectly competitive.

For $0 \leq x \leq 1$, define

$$
\alpha(x) \equiv \sum_{n=0}^{F} \alpha_{n} x^{n}, \quad \beta(x) \equiv \sum_{n=0}^{F} \beta_{n} x^{n}
$$

to be the respective probability generating functions for the number of prices observed by the two kinds of consumer. Suppose that the number of prices observed by the more informed consumers (first order) stochastically dominates the number observed by the less informed consumers, which can be shown to imply $\alpha(x) \leq \beta(x)$. Suppose a fraction $\lambda$ of consumers are informed. (We will discuss shortly how $\lambda$ is determined.) Let $\varphi(x)=\lambda \alpha(x)+(1-\lambda) \beta(x)$, and let $\varphi_{n}=\lambda \alpha_{n}+(1-\lambda) \beta_{n}$ be the proportion of all consumers who see $n$ prices.

How do firms set prices when faced with this population of consumers? The answer is given by an extension to Burdett and Judd's (1983) analysis. There is a symmetric mixed strategy equilibrium in which each firm chooses a price greater than $p$ with probability $x(p)$ on the support $\left[p_{L}, v\right]$. The proportion of consumers who see $i$ 's price and who also see exactly $n-1$ other prices is $n \varphi_{n} / F$. A consumer will buy from firm $i$ if she sees $i$ 's price and that price is lower than any other price she observes, and so $i$ 's expected demand with price $p$ is

$$
\frac{\varphi_{1}+2 \varphi_{2} x(p)+3 \varphi_{3}(x(p))^{2}+\ldots}{F}=\frac{\varphi^{\prime}(x(p))}{F} .
$$


Because each firm must be indifferent between choosing all prices in the support $\left[p_{L}, v\right]$, and because $v$ is in this support, we have

$$
p \frac{\varphi^{\prime}(x(p))}{F} \equiv v \frac{\varphi^{\prime}(x(v))}{F}=v \frac{\varphi^{\prime}(0)}{F}=v \frac{(1-\lambda) \beta_{1}}{F} .
$$

In particular, industry profit, which is the expected price paid by a random consumer, is

$$
P \equiv v(1-\lambda) \beta_{1}
$$

Expression (2) implies that all $p$ in the support satisfy $p \varphi^{\prime}(x(p)) \equiv P$, which implicitly defines the equilibrium choice of $x(\cdot)$. It is more convenient to use the inverse function $p(x)$ rather than $x(p)$, that is, $x(p(x)) \equiv x$, which in equilibrium satisfies

$$
p(x)=\frac{P}{\varphi^{\prime}(x)} .
$$

In particular the lowest price in the support is $p_{L}=p(1)$ in expression (4).

The density of the lowest of $n$ prices is

$$
\frac{d}{d p}\left(1-[x(p)]^{n}\right)=-n[x(p)]^{n-1} x^{\prime}(p),
$$

so the expected price paid by an informed consumer, $P_{I}$, is

$$
-\sum_{n=1}^{F} \int_{p_{L}}^{v} p \alpha_{n} n[x(p)]^{n-1} x^{\prime}(p) d p=-\int_{p_{L}}^{v} p \alpha^{\prime}(x(p)) x^{\prime}(p) d p=\int_{0}^{1} p(x) \alpha^{\prime}(x) d x .
$$

Here, the final equality follows from changing variables from $p$ to $x(p)$. From equation (4), an explicit formula for $P_{I}$ is

$$
P_{I}=P \int_{0}^{1} \frac{\alpha^{\prime}(x)}{\varphi^{\prime}(x)} d x
$$

Likewise, the expected price paid by an uninformed consumer, $P_{U}$, is

$$
P_{U}=P \int_{0}^{1} \frac{\beta^{\prime}(x)}{\varphi^{\prime}(x)} d x .
$$

Of course, the average price paid, $P$, is equal to $\lambda P_{I}+(1-\lambda) P_{U}$.

The gross benefit to a consumer of being informed is

$$
P_{U}-P_{I}=\int_{0}^{1} p(x)\left[\beta^{\prime}(x)-\alpha^{\prime}(x)\right] d x=\int_{0}^{1} p^{\prime}(x)[\alpha(x)-\beta(x)] d x \geq 0,
$$

where the second equality follows from integration by parts and the observation that $\alpha(0)=\beta(0)=0$ and $\alpha(1)=\beta(1)=1$, while the inequality follows from 
stochastic dominance. Note that when all consumers are informed $(\lambda=1)$, we have $P=P_{U}=P_{I}=0$. Thus the incentive to become informed is non-negative, and approaches zero when nearly all consumers are informed.

The following result summarizes further properties of these expected prices:

Lemma 1. (i) $P_{I}$ and $P_{U}$ decrease with $\lambda$, (ii) $P_{U}-P_{I}$ is strictly concave in $\lambda$, and (iii) $P_{I} / P_{U}$ decreases with $\lambda$.

Proof. Write $g \equiv \alpha^{\prime}(x) /\left[\lambda \alpha^{\prime}(x)+(1-\lambda) \beta^{\prime}(x)\right]$, in which case

$$
\frac{1-\lambda g}{1-\lambda}=\frac{\beta^{\prime}(x)}{\lambda \alpha^{\prime}(x)+(1-\lambda) \beta^{\prime}(x)} ; \quad g_{\lambda}=\frac{\partial g}{\partial \lambda}=\frac{g(1-g)}{1-\lambda} .
$$

(i) Differentiating equation (5) yields

$$
\frac{d P_{I}}{d \lambda}=\frac{d}{d \lambda} v \beta_{1}(1-\lambda) \int_{0}^{1} g d x=v \beta_{1} \int_{0}^{1}\left[(1-\lambda) g_{\lambda}-g\right] d x=-v \beta_{1} \int_{0}^{1} g^{2} d x<0 .
$$

Similarly, differentiating equation (6) yields

$$
\frac{d P_{U}}{d \lambda}=-v \beta_{1} \int_{0}^{1} g \frac{1-\lambda g}{1-\lambda} d x<0 .
$$

(ii) Noting that

$$
P_{U}-P_{I}=v \beta_{1} \int_{0}^{1}[1-g] d x,
$$

it follows that $P_{U}-P_{I}$ is strictly concave in $\lambda$ because $g$ is strictly convex in $\lambda$.

(iii) Using expressions (5)-(6) and differentiating with respect to $\lambda$ shows that $P_{I} / P_{U}$ decreases with $\lambda$ if

$$
\begin{aligned}
\left(\int_{0}^{1}[1-\lambda g] d x\right)\left(\int_{0}^{1}\left[(1-\lambda) g_{\lambda}-g\right] d x\right) \\
+\left(\int_{0}^{1}(1-\lambda) g d x\right)\left(\int_{0}^{1}\left[\lambda g_{\lambda}+g\right] d x\right)
\end{aligned}
$$

is negative. But this expression simplifies to

$$
\left(\int_{0}^{1} g d x\right)^{2}-\int_{0}^{1} g^{2} d x,
$$

which is indeed negative by the Cauchy-Schwarz Inequality. 
Part (iii) of this result states that the average price paid by an informed consumer falls proportionately more than that paid by an uninformed consumer when there is an increase in the number of informed consumers.

Suppose a consumer can choose to use the superior search technology by incurring a (possibly psychological) cost $s \geq 0$. In general, consumers may differ in their cost of acquiring information, and let $s(\lambda)$ be the information cost of the marginal consumer when $\lambda$ consumers choose to be informed. (The function $s(\cdot)$ is weakly increasing.) In addition, write $S(\lambda)=\int_{0}^{\lambda} s(\tilde{\lambda}) d \tilde{\lambda}$ for the total cost incurred when $\lambda$ consumers become informed. For the marginal consumer to be indifferent between being informed or uninformed, the fraction $\lambda$ of consumers who choose to become informed satisfies

$$
P_{U}(\lambda)-P_{I}(\lambda)=s(\lambda) .
$$

If information costs are so large that there is no solution to equation (8), then no consumer chooses to become informed and $\lambda=0$. (In this case, all consumers pay $P_{U}=P=\beta_{1} v$.) Without making further assumptions, it is possible that there are several solutions to equation (8). ${ }^{1}$ However, because $s$ is above $P_{U}-P_{I}$ for $\lambda$ close to 1 , if the two curves cross at all, at least one intersection $P_{U}-P_{I}$ will cross $s$ from above. (See Figure 1 for an illustration.) When there are several

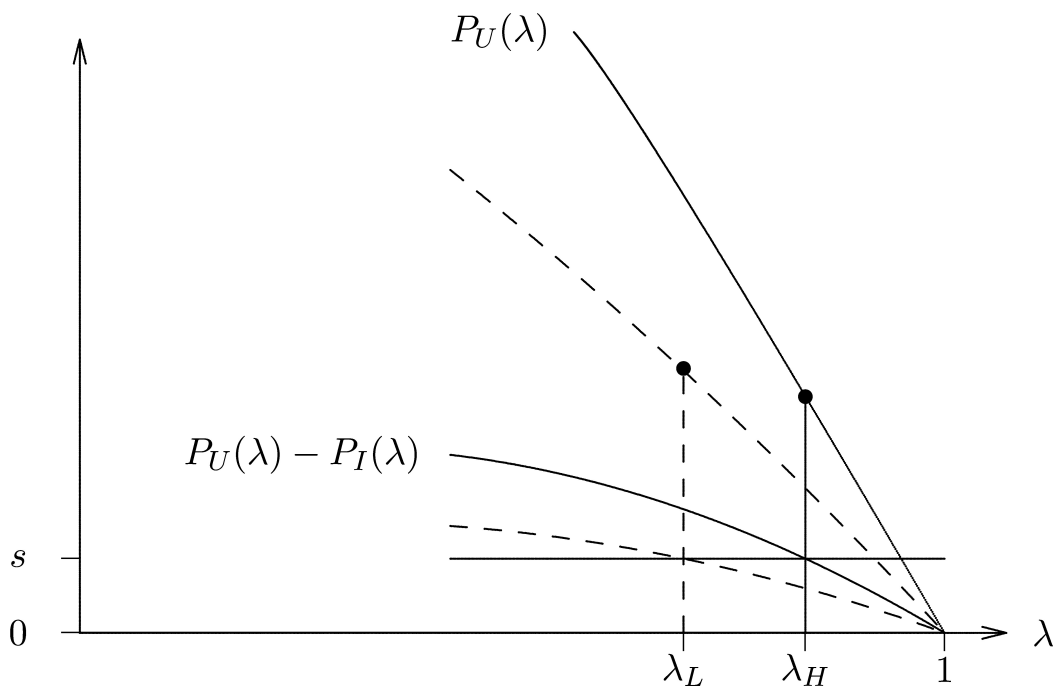

FIGURE 1. The impact of a price cap.

1. Because $P_{U}-P_{I}$ is concave in $\lambda$, in the special case where $s(\lambda)$ is constant, or convex, there can be at most two solutions to equation (8). 
roots to equation (8), we assume that a root where $P_{U}-P_{I}$ crosses $s$ from above is the selected equilibrium (because only these equilibria are stable).

As is often the case in search models, in this market there is too little information acquisition in equilibrium from the consumer viewpoint. Total outlay by consumers is $P+S(\lambda)$, where $P$ is the average price in equation (3). This total outlay is decreasing in $\lambda$ if $v \beta_{1}>s(\lambda)$, which is always the case whenever some consumers have an incentive to become informed. ${ }^{2}$

To illustrate this discussion, consider an example where uninformed consumers know just one price, so $\beta(x)=x$, and informed consumers know two prices, so $\alpha(x)=x^{2}$. (This example corresponds to the model in Burdett and Judd [1983] and Fershtman and Fishman [1994].) Then equations (5) and (6) imply

$$
P_{I}=v \frac{1-\lambda}{\lambda}\left(1-\frac{1-\lambda}{2 \lambda} \log \frac{1+\lambda}{1-\lambda}\right) ; \quad P_{U}=v \frac{1-\lambda}{2 \lambda} \log \frac{1+\lambda}{1-\lambda} .
$$

If all consumers have information cost $s=v / 20$, one can show numerically that approximately $95 \%$ of consumers become informed. All consumers make the expected payment (including information costs where relevant) of $P_{U} \approx v / 10$.

\section{Two Consumer Protection Policies}

\subsection{Imposing a Price Cap}

Consider a policy aimed at protecting uninformed consumers against unduly high prices. (For instance, a usury law might take this form, or consumer advocates might suggest such regulation in the energy or telecommunications sectors if some consumers are found to be paying high prices.) That is to say, policy constrains firms to set prices no higher than $\bar{p}$, where $\bar{p}<v$ is a market price cap. Then all the analysis in Section 2 remains valid so long as $v$ is replaced everywhere by $\bar{p}$. In particular, the expected prices paid by informed and uninformed consumers are now $(\bar{p} / v) P_{I}$ and $(\bar{p} / v) P_{U}$, respectively, where $P_{I}$ and $P_{U}$ are given in equations (5)-(6). A price cap has pros and cons. For given $\lambda$, the intervention benefits both the informed and the uninformed consumers because the prices they pay are proportional to $\bar{p}$. But the incentive to become informed, $(\bar{p} / v)\left(P_{U}-P_{I}\right)$, is also proportional to $\bar{p}$ for given $\lambda$, and so the cap causes the number of informed consumers to fall.

Consider imposing the price cap $\bar{p}=v / 2$ in the numerical example, so that maximum prices are halved. In this case the fraction of informed consumers satisfies $P_{U}(\lambda)-P_{I}(\lambda)=2 s$ which entails $\lambda \approx 0.74$. Thus, the fraction of uninformed consumers rises about 5-fold as a result of the cap. Each consumer

2. From equation (7), if in equilibrium $\lambda>0$ we must have $v \beta_{1} \int_{0}^{1}(1-g) d x=s(\lambda)$, and so $v \beta_{1}>s(\lambda)$. 
pays $(\bar{p} / v) P_{U}$, which is now increased by about $70 \%$ to $0.17 \times v$. Industry profit in equation (3) more than doubles as a result of the imposition of the price cap, rising from around $0.05 \times v$ without regulation to $0.13 \times v$. $^{3}$

Note that when the price cap is tight enough (in this example, this occurs when $\bar{p} / v$ is below around 0.48 ), there is no equilibrium in which any consumer chooses to become informed, and the regime shifts discontinuously to the Diamond Paradox in which all consumers shop randomly and firms price deterministically at the cap. (This feature is due to the fact that $\beta_{1}=1$ in this numerical example, so there is no price competition when all consumers are uninformed.) In this case, regulation entirely displaces competition as the market discipline.

Beyond this numerical example, when does imposing a price cap harm consumers? With a cap $\bar{p}$, aggregate consumer outlay is

$$
\frac{\bar{p}}{v}\left[\lambda P_{I}(\lambda)+(1-\lambda) P_{U}(\lambda)\right]+S(\lambda),
$$

where $\lambda$ satisfies $(\bar{p} / v)\left[P_{U}(\lambda)-P_{I}(\lambda)\right]=s(\lambda)$. Therefore, in equilibrium, total consumer outlay as a function of $\lambda$ is

$$
s(\lambda) \frac{P_{U}(\lambda)}{P_{U}(\lambda)-P_{I}(\lambda)}-\lambda s(\lambda)+S(\lambda) .
$$

Because $\lambda$ is an increasing function of $\bar{p}$ whenever some consumers search, consumer welfare increases with $\bar{p}$ when equation (9) decreases with $\lambda$. Differentiating expression (9) yields

$$
\frac{d}{d \lambda}\left[s \frac{P_{U}}{P_{U}-P_{I}}-\lambda s+S\right]=\underbrace{s \frac{d}{d \lambda} \frac{P_{U}}{P_{U}-P_{I}}}_{<0}+\underbrace{\left\{\frac{P_{U}}{P_{U}-P_{I}}-\lambda\right\}}_{\geq 0} s^{\prime} .
$$

The first term in equation (10) is negative from part (iii) of Lemma 1, and the second term is positive if $s(\lambda)$ is strictly increasing. In the special case where all consumers have the same search cost $\left(s^{\prime} \equiv 0\right)$, expression (10) is surely negative. Thus, as discussed in Fershtman and Fishman (1994), provided the price cap is not so tight that all consumers cease searching, the imposition of a price cap makes all consumers pay higher expected prices.

This analysis with homogenous search costs is illustrated in Figure 1 above. Without a price cap, the equilibrium number of consumers who become informed, $\lambda_{H}$ in the figure, is found where the $P_{U}-P_{I}$ curve meets the search cost curve

3. Note that total welfare (the sum of profit and consumer welfare) in this unit demand framework is simply $W=v-S(\lambda)$, which decreases with $\lambda$. Thus, total welfare here is improved by the price cap because of the moral hazard induced by regulation. If the model were extended slightly to elastic rather than unit demands, there would be a welfare loss induced by high prices, which might outweigh the benefits of reduced expenditure on information acquisition. 
$s$ (assumed flat in the figure), and the outlay of each consumer is then given by the price $P_{U}$ evaluated at $\lambda_{H}$. (Even informed consumers incur this outlay, because they are indifferent between being informed and not being informed.) If a cap is imposed, this causes both $P_{U}-P_{I}$ and $P_{U}$ to fall equi-proportionately, to the dashed curves on the figure. The result is that the informed fraction falls to $\lambda_{L}$, and the outlay of each consumer rises. The mathematical property which ensures that the indirect harm (moving higher up the $P_{U}$ curve) outweighs the direct benefit (moving to a lower $P_{U}$ curve) is precisely that $P_{I} / P_{U}$ falls with $\lambda$. Because $P_{U}-P_{I}$ is concave, it either monotonically decreases over the range $\lambda \in[0,1]$, or attains a maximum for some interior $\lambda$. In the latter case, when the price cap is made sufficiently tight that $P_{U}-P_{I}$ falls below the horizontal search line, all consumers will choose to remain uninformed and expected prices will jump discontinuously to the situation corresponding to $\lambda=0$.

Clearly, as the cap approaches marginal cost, equilibrium prices will converge to marginal cost and consumers benefit from the policy. More generally, if $P_{U}(\lambda)=P_{I}(\lambda)+s$ is each consumer's expected outlay without regulation (where $\lambda$ is the corresponding number of informed consumers), then any price cap $\bar{p} \leq P_{U}(\lambda)$ forces firms to set prices no higher than in the laissez-faire market, and the intervention will surely benefit all consumers. How tight does the cap have to be to benefit consumers? The previous discussion has shown that whenever the price cap is not so tight that all consumers choose to remain uninformed, the price cap raises expected consumer outlay. Therefore, for the cap to benefit consumers it is necessary (but not in general sufficient ${ }^{4}$ ) that it be so tight that all consumers choose to remain uninformed. When $\lambda=0$ each consumer pays the price $\beta_{1} \bar{p}$. Hence, the cap will benefit consumers if and only if

$$
\bar{p} \leq \frac{P_{U}(\lambda)}{\beta_{1}},
$$

where $P_{U}(\lambda)$ is an uninformed consumer's payment in the absence of regulation.

This discussion formalizes a claim sometimes made informally, which is that imposing price controls on an oligopoly market could act to raise equilibrium prices. One intuition for such a claim is that a price cap acts as a focal point for tacit collusion. Equilibria in this model, however, are non-cooperative, so tacit collusion plays no role. Rather, price controls soften competition by blunting consumers' incentives to search for good deals. Although the direct effect of a price cap is to reduce prices, the indirect effect of reduced search lessens each firm's demand elasticity so much that prices on average go up.

However, if consumers differ in their costs of acquiring information, imposing a price cap causes fewer consumers to cease becoming informed. If $s(\lambda)$ is

4. Recall from the numerical example that the price may jump upward at the point when regulation just removes all incentive to become informed. 
sufficiently steep, expression (9) increases with $\lambda$ and a price cap will then benefit consumers. Consider the limit case where an exogenous fraction of consumers $\lambda$ are informed while the remaining consumers are uninformed. This situation could be interpreted as there being a fraction $\lambda$ of consumers who have zero search cost and the remainder have an infinite search cost; or we could hold a behavioral interpretation, that a fraction $1-\lambda$ of consumers are "naive" and mistakenly believe there is no benefit to shopping around. When $\lambda$ is constant, the imposition of a price cap is unambiguously beneficial for both groups of consumers, and harms industry profits. In intermediate cases, an upward-sloping curve representing search costs on Figure 1 (as opposed to a vertical or horizontal line) makes the net impact of a price cap ambiguous for consumers.

\subsection{Enabling Consumers to Opt Out of Advertising}

Nowadays consumers have various means by which to limit the volume of marketing materials they receive. ${ }^{5}$ Television recording devices allow consumers to skip through advertising breaks, and consumers on the Internet can use pop-up blockers and spam filters to lessen intrusive advertising. A popular consumer policy is to introduce a "do not call" list, to which consumers can sign up and choose not to receive telemarketing from firms. Those consumers whose costs of receiving marketing outweigh the benefits from learning about the deals available in the market will therefore choose to avoid ads. We can think of those consumers who refuse to receive ads as constituting the uninformed pool of consumers, and those who remain willing to receive marketing are the more informed.

In more detail, consider a policy which enables consumers to refuse to accept advertising by signing up to a list. Suppose for simplicity that firms can costlessly attempt to send ads to consumers who are not on the list (or to all consumers if no such list is introduced). Consumers who do not sign up to the list will be informed, namely, they will incur their marketing disutility $s$ and obtain the random number of price observations governed by $\alpha(\cdot)$. Those consumers who choose not to receive marketing will be less informed - they will see the number of prices govern by $\beta(\cdot)$-but avoid the disutility $s$. Assume that firms cannot price discriminate according to whether a consumer has signed up to the list.

When no such list is introduced, consumers are assumed to have no method to avoid adverts and all consumers will be informed $(\lambda=1)$. By assumption (1), the market will then be perfectly competitive. Therefore, all consumers will pay a price equal to marginal cost but in aggregate they will incur advertising disutility

5. There is a substantial literature discussing the impact of consumer ad-avoidance. See for example Hann et al. (2008) and the references therein for discussion. However, most of this literature makes the simplifying assumption that prices are exogenously fixed. Such papers are unable to address the issue of how ad-avoidance affects price competition. 
$S(1)$. When the list is introduced and $1-\lambda$ consumers choose to sign up, firms will price as described in Section 2. The equilibrium fraction of consumers who sign up satisfies condition (8). (If disutilities are so large that no solution to (8) exists, then all consumers sign up and $\lambda=0$.) As discussed in Section 2 , from a consumer point of view too many consumers sign up to the list. ${ }^{6}$ If the list is abandoned, this forces all consumers to become informed, which moves $\lambda$ in the right direction but with the danger that the correction goes too far.

In more detail, after the list is introduced total outlay by consumers is

$$
\lambda P_{I}(\lambda)+(1-\lambda) P_{U}(\lambda)+S(\lambda)=P_{I}(\lambda)+(1-\lambda) s(\lambda)+S(\lambda),
$$

and so the increase in total outlay due to the introduction of the list is

$$
\underbrace{P_{I}(\lambda)}_{>0}+\underbrace{(1-\lambda) s(\lambda)-[S(1)-S(\lambda)]}_{\leq 0} .
$$

The first term is clearly positive (unless no one signs up to the list), and the second term is non-positive because $s(\cdot)$ is weakly increasing. As in Section 3.1, the comparison is clear-cut in the special case where all consumers have the same cost $s$. Here, the second term in equation (12) vanishes, and the introduction of the list causes all consumers to be made worse off. In economic terms, without the "do not call" list all consumers incur disutility $s$ but obtain the product at marginal cost. When the list is introduced, all consumers pay the informed consumers' price, which is $s$ plus an imperfectly competitive price $P_{I}$ above marginal cost. When a consumer decides based on her private cost-benefit calculation to sign up to the list, this reduces the fraction of informed consumers, which in turn harms all consumers via the higher prices which then ensue. Moreover, profits-which from expression (3) are negatively related to the number of informed consumers-rise when the list is introduced. Thus, firms may support the introduction of "do not call" lists and the like, for the same reason that firms historically have sometimes supported measures to restrict price advertising. ${ }^{7}$

When some consumers have higher marketing disutility than others, though, the comparison is ambiguous. In the case where some consumers are extremely averse to receiving unsolicited marketing, the list will enhance aggregate consumer welfare. However, there is then a distributional effect: Those consumers who are not strongly ad-averse (i.e., those who do not sign up to the list when it is introduced) will be harmed by the policy, because the price they pay rises due to the decreased consumer monitoring.

6. Anderson and de Palma (2008) study a model in which firms do not compete in prices and where consumers dislike seeing ads. They discuss a "do not call" list, and find that too many consumers sign up to such a list from the viewpoint of total welfare (not consumer welfare). The cause is not externalities between consumers (as in our model), but the fact that consumers ignore the negative impact their opt-out decision has on supplier profits.

7. See, for instance, Armstrong (2008, Section V) for further discussion. 


\section{Possible Extensions}

This paper has discussed some possibly undesirable effects of consumer protection policies. Using a parsimonious oligopoly model with price dispersion, we argued that (i) imposing a price cap might lead to price rises to consumers and (ii) permitting consumers to opt out of advertising might make all consumers worse off. In each case, the cause of the harm was that the policy reduced the number of informed consumers, and the resulting weakening of competitive pressure led firms to charge higher prices.

It would be useful to extend this stylized model to richer settings. For instance, it is not common to impose caps on headline prices in oligopoly markets, as we assumed in Section 3.1. Rather, price controls might be applied to "small print" charges in a contract, or minimum quality standards might be imposed on aspects of product quality. It would be worthwhile to extend our model so that consumers must expend effort to understand these less salient aspects of a firm's offer. For instance, could the introduction of a minimum quality standard sometimes lead to lower average quality in the market?

\section{References}

Anderson, Simon, and André de Palma (2008). "Information Congestion: Open Access in a Two-Sided Market." Working paper, University of Virginia.

Armstrong, Mark (2008). "Interactions between Competition and Consumer Policy." Competition Policy International, 4, 97-147.

Burdett, Kenneth, and Kenneth Judd (1983). "Equilibrium Price Dispersion.” Econometrica, 51, 955-969.

Fershtman, Chaim, and Arthur Fishman (1994). “The 'Perverse' Effects of Wage and Price Controls in Search Markets." European Economic Review, 38, 1099-1112.

Hann, Il-Horn, Kai-Lung Hui, Sang-Yong Lee, and Ivan Png (2008). "Consumer Privacy and Marketing Avoidance: A Static Model.” Management Science, 54, 1094-1103. 